\title{
Clinical Spectrum of COVID-19 in Children at a Tertiary Care Centre
}

\author{
Ankur Joshi ${ }^{1}$, Gunjan Kela ${ }^{2}$, Nandini Sodha ${ }^{3}$, Pragya Mehta ${ }^{4}$ \\ 1,2,3,4 Department of Paediatrics, Sri Aurobindo Medical College and Post Graduate Institute, \\ Indore, Madhya Pradesh, India.
}

\section{INTRODUCTION}

Increasing cases of COVID-19 have been reported in children globally. Admission frequency, though, is significantly lower than adults in COVID-19. Recent evidence suggests that children likely have the same or higher viral loads. Our study is retrospective, with the purpose to describe the clinical spectrum of this disease in children and response of treatments, along with its effect in the neonatal period. As very few cases have been encountered with severe disease, and are illustrated in this study, attempting to understand the course of severe illness.

Globally and, as compared with adults, fewer cases of COVID-19 have been reported in children (age 0 - 17 years).1,2 Hospitalization rates in children are significantly lower than hospitalization rates in adults with COVID-19, suggesting that children may have a less severe illness from COVID - 19 compared to adults. ${ }^{3,4}$ Recent evidence suggests that children likely have the same or higher viral loads in their nasopharynx compared with adults,, 5 and those children can spread the virus effectively in households and camp settings. 7,8

At our institute, $10 \%$ of the total COVID-19 cases were from the paediatric population. Among the early neonatal period following delivery to a COVID positive mother, most of them were FT deliveries (83\%), with the majority delivered vaginally. In relation to PT cases, all were delivered vaginally including 4 cases of documented intrauterine device (IUD) by induction of labour with a zero transplacental transmission rate. Out of positive cases in the perinatal period, all were presented as necrotizing enterocolitis (NEC). In the paediatric age group of 1 month - 17 years, varied symptoms were present. Children having comorbidities contributed to poor prognosis.
Corresponding Author:

Dr. Gunjan Kela,

Department of Paediatrics,

Sri Aurobindo Medical College and

Post Graduate Institute, Indore,

Madhya Pradesh, India.

E-mail:drgunjankela@yahoo.com

DOI: $10.14260 / j e m d s / 2021 / 754$

How to Cite This Article:

Joshi A, Kela G, Sodha N, et al. Clinical spectrum of covid-19 in children at a tertiary care centre. J Evolution Med Dent Sci 2021;10(43):3726-3729, DOI: 10.14260/jemds/2021/754

Submission 16-05-2021,

Peer Review 04-09-2021

Acceptance 11-09-2021,

Published 25-10-2021.

Copyright (c) 2021 Ankur Joshi et al. This is an open access article distributed under Creative Commons Attribution License [Attribution 4.0 International (CC BY 4.0)] 


\section{PRESENTATION OF CASE}

We have admitted over 600 cases of COVID-19 in the paediatric age group, including neonatal cases. Out of which, a few severe cases with varied presentation are illustrated below, requiring intensive care.

\section{Paediatric Age Group 1 month - 17 years}

1. A 15 yr old female came with a complaint of haemoptysis and chest pain, HRCT chest gave ground-glass attenuation suggestive of alveolitis (2 - 4 \%). COVID-19 RT - PCR came positive, and the child responded well to HCQS, steroids and symptomatic treatment. There was no history of contact with COVID-19.

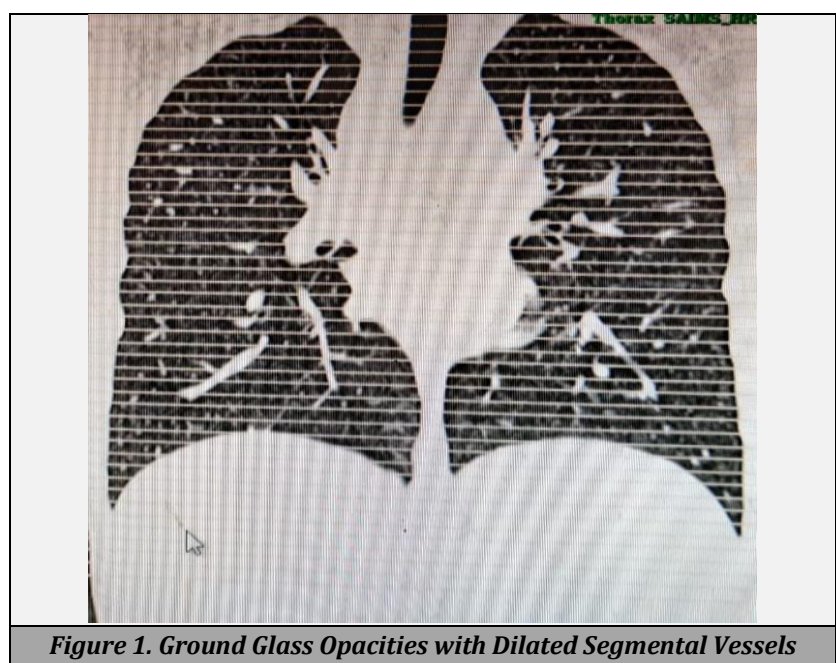

2. A 13 year old female was admitted with fever and signs of congestive cardiac failure. 2D-echo revealed myocarditis, child improved well on steroids and intravenous immunoglobulin (IVIG). RT - PCR for COVID19 was positive. The history of contact was negative.

3. A 13 year old female child was admitted with bicytopenia (anaemia and thrombocytopenia) and positive for malarial antigen and peripheral smear was reported with $P$. vivax.

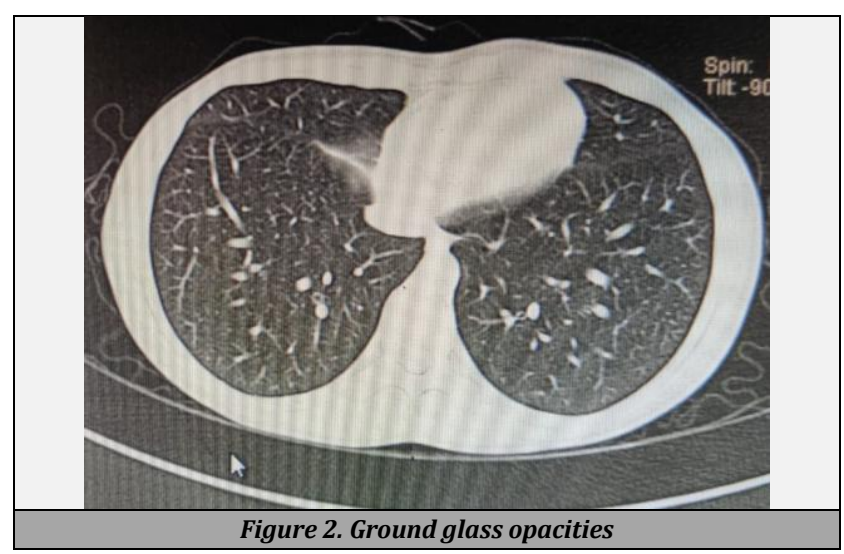

4. For persistent fever, the child was evaluated for COVID19 as she had a contact history with COVID-19. Chest Xray revealed bilateral homogeneous opacities and bilateral pleural effusion. COVID-19 RT - PCR came positive. Due to the financial constraints of parents, treatment could not be continued at the institute. After discharge, the child lost to follow up.

5. 11 year old female, with a history of an operated case of acyanotic CHD (VSD) at 7 years of age, came with acute abdomen, dyspnoea and multiple organ dysfunction syndrome (MODS). The stay of the child was only $9-10$ hours at our institute. CT chest and abdomen suggested ground-glass attenuation with fibrotic changes in both lungs, along with moderate pleural effusion and ascites (CORAD 5). The patient outcome was fatal because of, multi-organ dysfunction and acute respiratory distress syndrome (ARDS).

6. A 7 year old female child came with meningitis and acute cervical lymphadenitis. CT chest revealed 40 - $45 \%$ lung involvement as ground-glass opacities, suggestive of COVID-19 infection with CORAD score 5 (CT score 12 / 25). USG neck local region findings suggestive of tubercular aetiology, diagnosis confirmed with FNAC. In this case, the child had a tubercular infection-causing immunocompromised state and hence developed COVID -19 as a co-infection. The child recovered from COVID19 uneventfully.

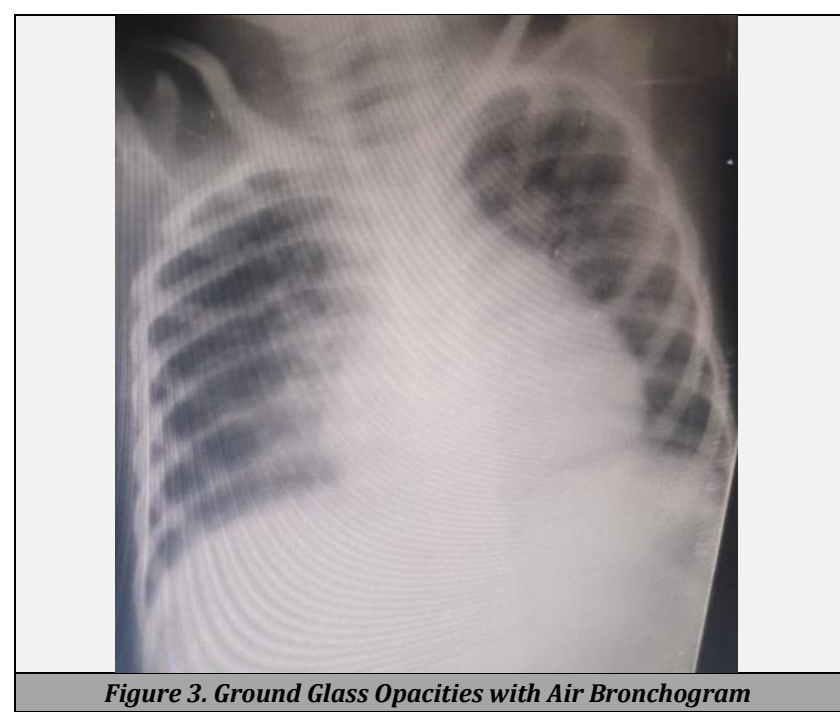

7. A 5-month-old undernourished male child was admitted with acute respiratory illness and breathing difficulty without any history of contact. $40 \%$ lung involvement presented on CT chest as ground-glass appearance probably due to COVID-19(CORAD - 5; CT score 7 / 25). RT - PCR came positive later. The child showed improvement on remdesivir.

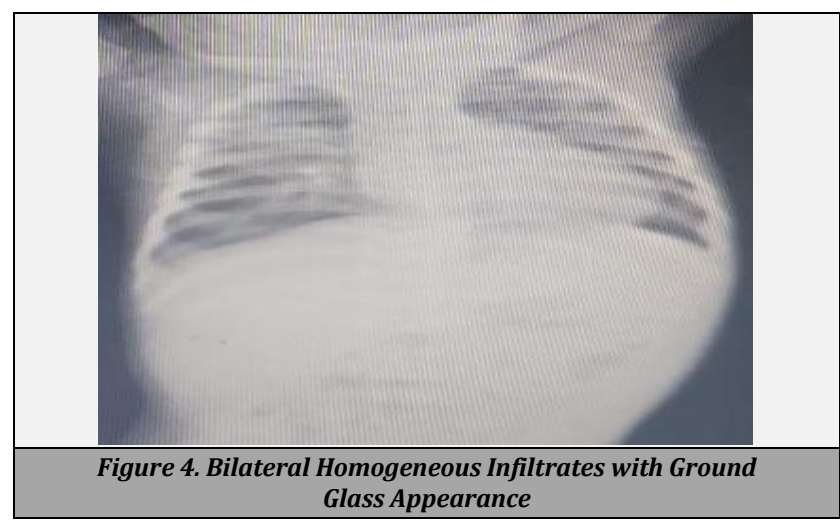


8. A 9 year old obese girl was admitted with a complaint of fever and lung involvement of $40 \%$ on CT chest. Child treated with hydroxychloroquine, doxycycline, and symptomatic treatment. The child had a positive history of contact with COVID-19, so RT - PCR was sent which came positive. She responded well to treatment.

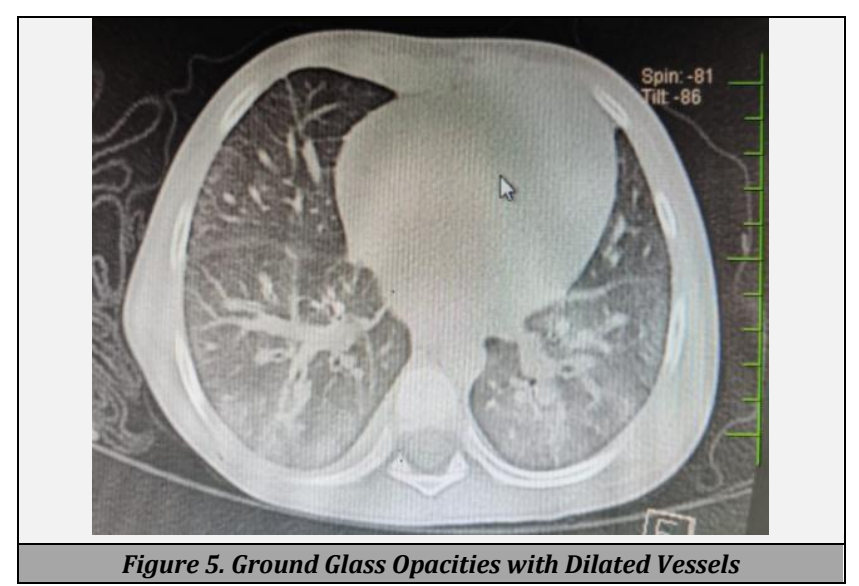

9. A 15 year old female was admitted for acute febrile illness, pain in abdomen and breathlessness, with $>70 \%$ lung involvement on CT chest (CORAD 5; CT score 19 / $25)$. The child required NIV support and aggressive management with remdesivir and tocilizumab (in view of cytokine storm). The patient dramatically improved over 48 hours and was discharged successfully after a hospital stay of 10 days.

10. A 13-year-old male child who came with congestive cardiac failure developed features of mild Guillain Barre Syndrome (GBS) during the hospital stay. COVID IgG antibody titre came positive (raised titres). The diagnosis was kept post COVID mild GBS and planned for IVIG. The child was required to prolong hospital stay, and improve on conservative treatment.

\section{Neonatal Cases}

In our institute, we have encountered 45 neonatal cases. Out of these cases, 43 were observed in the early neonatal period and 2 in the late neonatal period.

1. A 17-day old female baby having a history of contact with COVID-19 came with a complaint of loose stool and abdominal distension, with the impression of NEC. RT PCR came positive for COVID. The child managed on the line of NEC improved on conservative treatment.

2. A male child was admitted on the $15^{\text {th }}$ day of life with COVID positive report, and $\mathrm{h} / \mathrm{o}$ twin preterm delivery, low birth weight and late-onset sepsis. The patient had multiple problems in the form of acyanotic CHD, fused vertebra, twin preterm delivery and low birth weight, contributing to grave disease. The child had B / L pneumonia with NEC, for which the child was aggressively managed. Even remdesivir was given along with ventilatory support. But, the outcome was fatal, and the child could not be saved.

\section{DISCUSSION}

The incubation period for the viral illness was found to be about 2 - 14 days with an average of 6 days, the same as in adults. ${ }^{9}$ Signs or symptoms of COVID-19 in children at the time of presentation are constitutional like any other viral illness with involvement of the upper and lower respiratory tract, GI system. New loss of taste or smell is typical for COVID in the current scenario. Sometimes it may present as asymptomatic. The most common symptoms in children are cough and / or fever.9,10,11,12,13 Evidence suggests that as many as $45 \%$ of paediatric infections are "asymptomatic." At our institute, among the total patients admitted with COVID 19 infections, $10 \%$ constituted the paediatric population. The number and rate of cases in children have been steadily increasing from March to September 2020. Among the early neonatal period following delivery to a COVID positive mother, most of them were FT deliveries (83\%), of which 46 $\%$ were delivered vaginally with spontaneous labour and 37 $\%$ via LSCS for an obstetric indication. In the case of PT cases, all were delivered vaginally including 4 cases of documented IUD by induction of labour. All live babies, whether FT or PT, were healthy at birth requiring only routine care, and had negative COVID-19 RT - PCR at 48 hours of life. This gave a zero trans-placental transmission rate. At our institute, we roomed in babies with their mothers with a mother wearing a mask and face shield. Out of positive cases in the perinatal period, all were presented as NEC. In the paediatric age group of 1 month - 17 years, varied symptoms were present. Among patients requiring intensive care, $24 \%$ had isolated organ involvement (myocarditis in $12 \%$, lung involvement in $12 \%$ ) and, the rest had multiple organ involvement. Children having comorbidities as an immune-compromised state [(HIV, TB), co-infection (Dengue, malaria), malnutrition, underlying illness (sickle cell disease, congenital heart disease)] contributed to poor prognosis. So, current evidence suggests, that similar to adults children with medical complexity might be at increased risk for severe illness from COVID-19.

\section{CONCLUSIONS}

The signs and symptoms of COVID-19 in children are similar to other infections and non - infectious processes, including influenza, streptococcal pharyngitis, and allergic rhinitis. The lack of specificity of signs or symptoms and the significant proportion of asymptomatic infections make symptom-based screening for identification of COVID-19 in children particularly "challenging." While children infected with COVID-19 are less likely to develop severe illness compared with adults, children are still at risk of developing severe illness and complications from COVID-19.14 There is limited evidence about which underlying medical condition in children might increase the risk for severe illness.

Financial or other competing interests: None.

Disclosure forms provided by the authors are available with the full text of this article at jemds.com. 


\section{REFERENCES}

[1] Stokes EK, Zambrano LD, Anderson KN, et al. Coronavirus Disease 2019 Case Surveillance-United States. January MMWR 2020;69(24):759-65.

[2] Wu Z, McGoogan JM. Characteristics of and important lessons from the coronavirus disease 2019 (COVID - 19) outbreak in China: summary of a report of 72314 cases from the Chinese center for disease control and prevention. JAMA 2020;323(13):1239-42.

[3] CDC COVID-19 Response Team. Coronavirus Disease 2019 in Children-United States, February 12-April 2, 2020. MMWR Morbidity and Mortality Weekly Report. 2020;69(14):422-6.

[4] Sargent TH, Muller WJ, Zheng X, et al. Age-related differences in nasopharyngeal severe acute respiratory syndrome coronavirus 2 (SARS - CoV - 2) levels in patients with mild to moderate coronavirus disease 2019 (COVID - 19). JAMA Pediatr 2020;174(9):902-3.

[5] Park YJ, Chloe YJ, Park O, et al. Contact tracing during coronavirus disease outbreak, South Korea, 2020. Emerg Infect Dis 2020;26(10):2465-8.

[6] Szablewski CM, Chang KT, Brown MM, et al. SARS-CoV-2 transmission and infection among attendees of an overnight camp - Georgia, June. 2020. MMWR 2020;69(31):1023-5

[7] Dong Y, Mo X, Hu Y, et al. Epidemiological characteristics of 2143 pediatric patients with 2019 coronavirus disease in China. Pediatrics 2020. DOI: 10.1542/peds.2020-0702
[8] Foster CE, Moulton EA, Munoz FM, et al. Coronavirus Disease 2019 in children cared for at Texas children's hospital: initial clinical characteristics and outcomes. J Pediatric Infect Dis Soc 2020;9(3):373-7.

[9] Assaker R, Colas AE, Julien-Marsollier F, et al. Presenting symptoms of COVID - 19 in children: a meta-analysis of published studies. Br J Anaesth 2020;125(3):e330-2.

[10] Boulad F, Kamboj M, Bouvier N, et al. COVID-19 in children with cancer in New York City. JAMA Oncol 2020;6(9):1459-60.

[11] Shekerdemian LS, Mahmood NR, Wolfe KK, et al. Characteristics and outcomes of children with coronavirus disease 2019 (COVID - 19) infection admitted to US and Canadian pediatric intensive care units. JAMA Pediatr 2020;174(9):868-73.

[12] Mannheim J, Gretsch S, Layden JE, et al. Characteristics of Hospitalized Pediatric COVID - 19 Cases - Chicago, Illinois. March - April 2020. J Pediatric Infect Dis Soc 2020;9(5):519-22.

[13] Poline J, Gaschignard J, Leblanc C, et al Systematic severe acute respiratory syndrome coronavirus 2 screening at hospital admission in children: a french prospective multicenter study. Clin Infect Dis 2021;72(12):2215-7.

[14] Xu H, Liu E, Xie J, et al. A follow up study of children infected with SARS-CoV-2 from Western China. Ann Transl Med 2020;8(10):623. 